\title{
Topical $0.05 \%$ Cyclosporine A Eyedrops for Treating Moderate-to-Severe Vernal Keratoconjunctivitis
}

\author{
Kenan ÇALIŞIR ${ }^{1}$, Taha AYYILDIZ², Ümit ÇALLI ${ }^{3}$
}

${ }^{1}$ Department of Ophthalmology, Horosan Public Hospital, Erzurum, Turkey.

${ }^{2}$ Department of Ophthalmology, Oltu Public Hospital, Erzurum, Turkey.

${ }^{3}$ Department of Ophthalmology, Umraniye Training and Research Hospital, İstanbul, Turkey.

\section{ABSTRACT}

This study aimed to investigate the efficacy of topical cyclosporine A (CSA) $0.05 \%$ in patients with moderate-to-severe vernal keratoconjunctivitis (VKC).

This retrospective study included 37 patients who were admitted to the clinic between January 2015 and December 2015. All patients were diagnosed with moderate-to-severe VKC and treated with topical CSA $0.05 \%$ (Restasis Allergan, Inc. CA, USA). The VKC diagnosis was based on patient history and typical clinical signs and symptoms. Sign and symptom scores of each patient were obtained at pretreatment and during treatment (1,3, and 6 months).

Of the 37 patients, 20 (54.1\%) were male and 17 (45.9\%) were female. The mean age of the patients was $11.83 \pm 4.70$ years (6-24 years). The symptom-sign and total scores of the patients were significantly reduced at 1, 3, and 6 months compared with baseline values. Symptom-sign scores also significantly reduced from 1 month to 3 months and from 3 months to 6 months $(P<0.05)$.

Topical CSA $0.05 \%$ is an effective formulation used for treating moderate-to-severe VKC. It may reduce the need for steroids.

Key Words: Cyclosporine A, topical, vernal keratoconjunctivitis

\section{INTRODUCTION}

Vernal keratoconjunctivitis (VKC) is a chronic, bilateral, conjunctival, inflammatory disease that develops as an atopic condition. The disease onset usually occurs before the age of 10 years, lasts for 2-10 years, and terminates around late puberty. VKC is more frequent in young men, whereas male/female ratio becomes comparable in older adults. The disease is bilateral in $96.7 \%$ of the patients, and all the unilateral cases are tarsal-form VKC $(1,2)$. In the beginning, $23 \%$ of the cases are perennial form, and more than 60\% show recurrence during winter (3). The disease occurrence increases during spring and summer, whereas it decreases during winter time in Mediterranean and other warm climate regions (4).

The pathogenesis of the disease has not been completely understood. In patients with VKC, high amounts of mast and eosinophil cell conglomerates were found in biopsy materials taken from the tarsal conjunctival papillary epithelium $(5,6)$. Specific immunoglobulin $\mathrm{E}(\mathrm{lg} E)$ and $\lg \mathrm{G}$ were found in tear samples of patients $(7,8)$. Histamine and tryptase also increased in tear samples of patients $(9,10)$. As VKC is an allergic condition related to lgE and helper T cell 2 (Th-2), it would be wrong to define it as a simple type 1 hypersensitivity reaction in the light of aforementioned considerations.

As in other atopic conditions, VKC treatment starts with avoiding the allergen. Short-term topical steroid treatment is effective in subsiding the symptoms of patients with a significant seasonal exacerbation. This treatment should be decreased as soon as possible to remove the symptoms of the disease. It has been known that long-term steroid use may lead to cataract and glaucoma. Therefore, additional measures should be taken to maintain the reduced inflammatory status achieved by short-term steroid treatment in these patients. During exacerbation, besides short-term topical steroid treatment, a mast cell stabilizer or mast cell stabilizer/antihistamine drugs such as olopatadine, ketotifen, epinastine, and azelastine may be started. 
TABLE 1: Symptom-sign scoring.

\begin{tabular}{lllll}
\hline Symptom - Sign & 0 & 1 & 2 & 3 \\
\hline Itching & None & Rare & Frequent & Always \\
Watery eye & None & Mild & Moderate & Severe \\
Photophobia & None & Mild & Moderate & Severe \\
Sense of foreign body & None & Mild & Moderate & Severe \\
Bulbar conjunctival hyperemia & None & Mild & Moderate & Severe \\
Tarsal papillary hypertrophy & None & Mild & Moderate & Severe \\
Punctate keratitis & None & Mild spotty & Diffuse spotty & Shield Ulcer \\
Corneal neovascularization & None & 1 quadrant & 2 quadrant & 3 quadrant \\
\hline
\end{tabular}

Topical cyclosporine (Cs) is a nonsteroid immunomodulator used for many ocular inflammatory diseases including VKC. Cs inhibits T lymphocyte activation and apoptosis in a group of cells $(9,10)$.

This study aimed to investigate the efficacy of topical Cs in the treatment of patients with moderate-to-severe VKC to prevent chronic steroid administration.

\section{MATERIALS AND METHODS}

This retrospective study investigated 37 patients diagnosed with moderate-to-severe VKC who were admitted to the clinic between January 2015 and December 2015. All patients were treated with topical CsA $0.05 \%$. The study was conducted in accordance with the Helsinki declaration. Consent was obtained from patients and their guardians. The VKC diagnosis was based on patient history, typical clinical symptoms, and signs. After taking the history of patients, detailed ophthalmologic examinations were also performed. Patients who were on systemic or topical immune suppressive treatment were excluded. Patients with any sign or symptom of blepharitis, dry eye, or viral or bacterial conjunctivitis were also excluded. Symptom and sign scorings were made at baseline and during treatment (1, 3, and 6 months). Clinical scores ranged between 0 and $3(0$, no sign or symptom of the disease; 3 , presence of severe symptoms or signs of the disease) (Table 1). A total score of 5 or more for each eye was accepted as severe VKC, whereas a score between 3 and 5 was moderate VKC.

Two weeks before treatment, topical antihistamines and/or mast cell stabilizers were stopped. One drop of topical CsA 0.05\% was applied 4 times a day to 37 patients with moderate-to-severe VKC. Data collected from patients were inputted into SPSS for Windows version 15 (SPSS, IL, USA). The homogeneity of distribution was tested by the Kolmogorov-Smirnov test. Comparisons were made using the paired t test. A P value less than 0.05 was considered significant.

\section{RESULTS}

The mean age of the patients was $11.83 \pm 4.70$ years ( $6-24$ years). The characteristics of the patients are demonstrated in Table 2. None of the patients had any other allergic pathologies except VKC. One patient had bilateral keratoconus (KC) and underwent penetrating keratoplasty for both eyes 4 years ago.

The mean symptom-sign and total scores of patients at baseline and at 1, 3, and 6 months of topical CsA treatment are given in Figure 1. Score analyses are shown in Table 3. A significant decrease in symptom-sign and total scores was found at 1, 3, and 6 months with respect to baseline values. Symptom-sign scores also significantly reduced from 1 month to 3 months and from 3 months to 6 months $(P<0.05)$.

TABLE 2: Demographic characteristics of the patients.

\begin{tabular}{|c|c|c|c|c|}
\hline $\begin{array}{l}\text { Age } \\
(N=37)\end{array}$ & $\begin{array}{l}\text { Gender } \\
(\mathrm{N}=37)\end{array}$ & $\begin{array}{l}\text { Limbal Type VKC } \\
(\mathrm{N}=16)\end{array}$ & $\begin{array}{l}\text { Papillary Type VKC } \\
(\mathrm{N}=7)\end{array}$ & $\begin{array}{l}\text { Mixed Type VKC } \\
(\mathrm{N}=14)\end{array}$ \\
\hline $\begin{array}{l}11.83 \pm 4.70 \\
(6-24 \text { Year })\end{array}$ & $\begin{array}{l}\text { Male }(\mathrm{N}=20) \text { : } \\
\% 54.1 \\
\text { Female }(\mathrm{N}=17) \text { : } \\
\% 45.9\end{array}$ & $\% 43.2$ & \% 18.9 & $\% 37.8$ \\
\hline
\end{tabular}




\begin{tabular}{|c|c|c|c|c|c|c|}
\hline $\begin{array}{l}\text { Follow-up } \\
\text { Period }\end{array}$ & $\begin{array}{l}\text { Symptom } \\
\text { score }\end{array}$ & $\begin{array}{l}\text { Sign } \\
\text { score }\end{array}$ & $\begin{array}{l}\text { Total } \\
\text { score }\end{array}$ & $\begin{array}{c}\text { Paired t test } \\
(P<0.05) \\
\text { symptom score }\end{array}$ & $\begin{array}{l}\text { Paired t test } \\
(P<0.05) \text { sign } \\
\text { score }\end{array}$ & $\begin{array}{l}\text { Paired t test } \\
(P<0.05) \text { total } \\
\text { score }\end{array}$ \\
\hline Pre-treatment & $8.56 \pm 1.96$ & $4.02 \pm 1.42$ & $12.62 \pm 2.75$ & & & \\
\hline 1. month & $6.21 \pm 2.58$ & $3.37 \pm 1.67$ & $9.56 \pm 3.68$ & & & \\
\hline 3. month & $3.54 \pm 2.04$ & $2.27 \pm 1.55$ & $5.86 \pm 3.01$ & & & \\
\hline 6. month & $1.14 \pm 1.11$ & $1.28 \pm 1.18$ & $2.32 \pm 2.02$ & & & \\
\hline Pre-treatment - 1. Month & & & & 0.017 & 0.004 & 0.011 \\
\hline Pre-treatment - 3. month & & & & 0.000 & 0.000 & 0.000 \\
\hline Pre-treatment -6. month & & & & 0.000 & 0.000 & 0.000 \\
\hline 1. month - 3. month & & & & 0.000 & 0.000 & 0.000 \\
\hline 1. month - 6. month & & & & 0.000 & 0.000 & 0.000 \\
\hline 3. month -6 . month & & & & 0.000 & 0.000 & 0.000 \\
\hline
\end{tabular}

During the follow-up period, third-month visits of four patients revealed a reduction in symptom-sign scores, and patient discomfort was evident. Therefore, topical loteprednol etabonate $0.5 \%$ (one drop four times a day, Bausch \& Lomb, Lotemax) was administered for 1 month to these patients. The patient discomfort gradually lowered. At the third-month visit, one of the patients was pregnant and her symptom-sign score was low. Therefore, the treatment continued with topical artificial tear drops (polyvinyl alcohol + povidone, Refresh single-use eyedrop, Allergan). One patient was referred to a higher-level center at the sixth-month visit due to persistent giant papillary structures on the left upper lid that compressed the cornea. That patient underwent cryoablation under general anesthesia. After treatment, it was revealed that stage 3 papillary structures regressed to stage 2 in the second week, and stage 2 punctate keratitis was abolished (stage 0). By the end of 6-month treatment, some of the patients completely recovered and drug treatment was stopped based on symptom-sign scores. Some other patients continued topical CsA treatment two times a day.

\section{DISCUSSION}

Topical cyclosporine $0.05 \%$ therapy was performed in this study four times a day for 6 months to treat patients with moderatesevere VKC. The patient group in the study had no steroid requirement and showed an improvement in the signs and symptoms of the disease.

VKC treatment is a difficult process for ophthalmologists because its pathogenesis is not clear and antiallergic treatments are usually not successful. Patients and their families should be

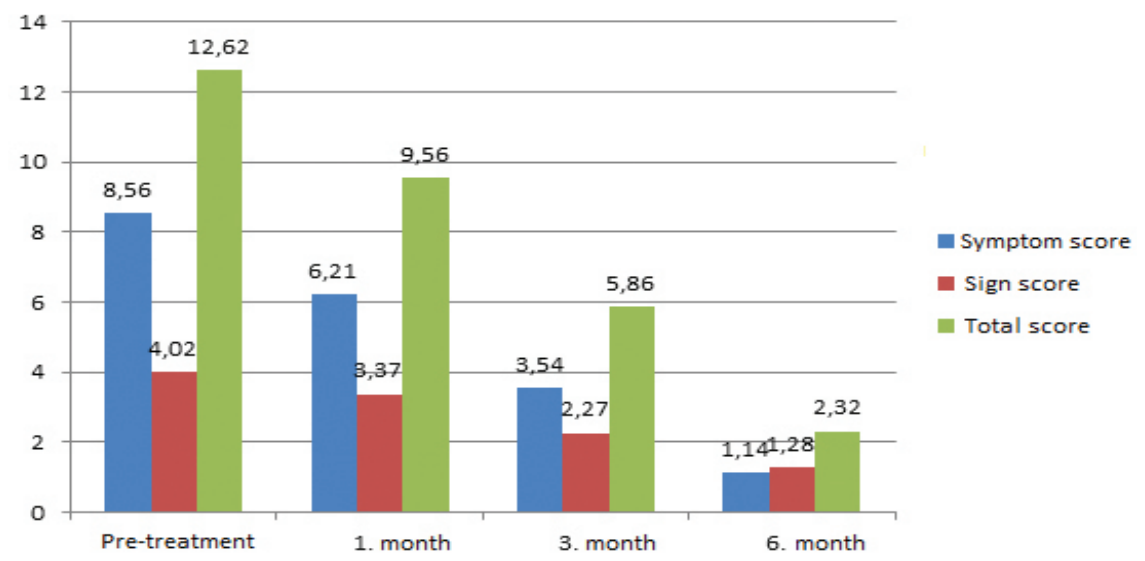

FIGURE 1: Symptom, sign, and total scores before and after treatment. 
informed about the nature, course, and possible complications of the disease. It is recommended that nonspecific triggering factors such as sunlight, wind, and salty water can be avoided using sunglasses or a hat.(11) The families were given this information at the beginning of treatment and recommended sunglasses and/or a hat.

Mast cell stabilizers and/or histamine receptor antagonists are mostly insufficient to control conjunctival inflammation and corneal involvement 13,14) Patients with moderate-to-severe VKC need recurrent topical steroid therapy to control conjunctival inflammation. However, corticosteroids should not be considered as the first-line treatment in VKC. If it is decided to administer corticosteroids, molecules with a lower intraocular absorption should be preferred (e.g., fluorometholone, loteprednol). The dosage should be adjusted based on the severity of inflammation in the eyes (12). Corticosteroids are not recommended for longterm use due to their side effects such as increase in intraocular pressure (IOP), onset of glaucoma or exacerbation, cataract formation, retarded wound healing, and tendency for infection or superinfection (15). In a case series reported from Singapore, 41 (28.3\%) of 145 patients with severe VKC showed corticosteroid response and 8 (5.5\%) of them progressed to glaucoma.(16) Topical loteprednol etabonate (one drop four times a day) was administered in this study to four patients at third-month visits, and the treatment was gradually stopped at the end of 1 month. No increase in IOP was observed in control visits of these patients.

CSA is effective in the control of VKC-related ocular inflammation through blocking Th-2 lymphocyte proliferation and interleukin (IL)-2 production. CSA inhibits histamine release from basophils and mast cells via a reduction in IL-5 production. Therefore, it may reduce eosinophil accumulation and action in the cornea and conjunctiva.(17) Although the formulation of $2 \%$ has the longest action, lower concentrations were also shown to be effective (1\%,0.5\%, and 0.05\%). No consensus exists till date on the minimal effective concentration of (SA. Only one commercially available formulation (0.05\%) is available for dry eye treatment. Itching and burning are common side effects. Treatment can be regulated on the seasonal or annual basis by reducing the dosage during inactive periods. (12) Increased serum levels of IL-17 and antinuclear antibodies, together with a high association with the familial history of autoimmune disorders, suggest the involvement of additional mechanisms in the development of VKC.
Çetinkaya et al. (18) reported that CsA 1\% was the minimum concentration in treating shield ulcer. Recurrence was reported with lower concentrations. A randomized controlled study showed that CSA $0.05 \%$ had effects similar to those of placebo (19). On the contrary, Özcan et al. (20) reported that CSA 0.05\% significantly reduced symptom severity and clinical findings with a 6-month treatment. It also reduced the need for steroids. Thus, low-dose CSA has been recommended as an escape agent from steroids in VKC treatment. In a randomized, placebo-controlled study, Keklikçi et al. (21) used topical CsA 0.05\% in treating patients with VKC and reported no side effects. Another study from Turkey showed that topical CSA 0.05\% significantly improved clinical symptoms and signs in patients with active VKC (22) A recent systematic review and meta-analysis has suggested that topical CsA leads to a significant improvement in sign and symptoms in a dose-independent manner and it is safe and effective in VKC treatment (23). This study has found a significant improvement in symptom-sign scores of patients with VKC treated with topical CSA $0.05 \%$ by the first month of treatment. Furthermore, symptom-sign scores also significantly reduced from 1 month to 3 months and from 3 months to 6 months.

The hydrophobic nature of the cyclosporine molecule, however, presented a challenge to developing an effective ophthalmic formulation. The poor water solubility of cyclosporine means that aqueous solutions at clinically useful concentrations are likely to be unstable. Attempts to overcome this have led to ophthalmic cyclosporine prepared in castor, corn, olive, and peanut oils. Burning, redness, itching, and epithelial keratitis limit the use of such oil vehicles (24). An emulsion formulation in castor oil, which also included glycerin, polysorbate 80 , and sodium hydroxide (to adjust the $\mathrm{pH}$ ), was developed (Restasis, Allergen, Inc., (A, USA) to improve the delivery of cyclosporine to ocular tissues (25). Upon instillation, cyclosporine partitions from the oil droplets in the emulsion into ocular surface tissues. Animal studies showed that topical administration of the emulsion formulation achieved cyclosporine concentrations sufficient for immunomodulation in the cornea and conjunctiva, but resulted in very low concentrations $(1 \mathrm{ng} / \mathrm{mL})$ in the aqueous humor, vitreous humor, and plasma. In this study, 0.05\% cyclosporine preparations with a water-based fat emulsion were used. No side effects were encountered in patients.

This study had certain limitations. First, it was a retrospective, observational study with noncontrolled groups. Second, no 
cytopathological assessment was made in this study. Finally, placebo was not included in the study. The disease could cause discomfort and sight-threating complications.

In conclusion, topical CsA $0.05 \%$ is an effective formulation for treating patients with moderate-to-severe VKC. It reduces the need for steroids. In patients who fail to achieve a significant improvement in symptom and signs, mildly effective topical corticosteroids can be added to the treatment for a short time.

\section{REFERENCES}

1. Leonardi A, Busca F, Motterle L, Cavarzeran F, Fregona IA, Plebani $M$ et all. Case series of 406 vernal keratoconjunctivitis patients: a demographic and epidemiological study. Acta Ophthalmol Scand 2006;84:406-410.

2. Keklikci U, Soker SI, Soker Cakmak S, Ozkul S, Sakalar YB, Unlu K. Unilateral vernal keratoconjunctivitis: A case report. Eur J Ophthalmol 2007;17:973-975.

3. Bonini $S$, Coassin M, Aronni $S$, Lambiase A. Vernal keratoconjunctivitis. Eye 2004;18: 345-351.

4. Pucci N, Massai C, Bernardini R, Caputo R, Mori F, De Libero C, et all. Eyelash length in children with vernal keratoconjunctivitis: effect of treatment with cyclosporine eye drops. Int J Immunopathol Pharmacol 2007;20:595-599.

5. Leonardi $A$, Sathe $S$, Bortolotti $M$, et al. Cytokines, matrix metalloproteases, angiogenic and growth factors in tears of normal subjects and vernal keratoconjunctivitis patients. Allergy 2009;64:710-7.

6. Zicari AM, Nebbioso M, Lollobrigida V, Bardanzellu F, Celani C, Occasi F, et al. Vernal keratoconjunctivitis: atopy and autoimmunity. Eur Rev Med Pharmacol Sci 2013;17:1419-23.

7. Zicari AM, Nebbioso M, Zicari A, Mari E, Celani C, Occasi $F$, et al. Serum levels of IL-17 in patients with vernal keratoconjunctivitis: a preliminary report. Eur Rev Med Pharmacol Sci 2013;17:1242-4.

8. Kumagai N, Fukuda K, Fujitsu Y, Yamamoto K, Nishida T. Role of structural cells of the cornea and conjunctiva in the pathogenesis of vernal keratoconjunctivitis. Prog Retin Eye Res 2006;25:165-87.

9. Utine CA, Stern M, Akpek EK. Clinical review: topical ophthalmic use of cyclosporin A. Ocul Immunol Inflamm 2010;18:352-61.

10. Halloran Lambiase A, Leonardi A, Sacchetti M, Deligianni V, Sposato S, Bonini S. Topical cyclosporine prevents seasonal recurrences of vernal keratoconjunctivitis in a randomized, double-masked, controlled 2-year study. J Allergy Clin Immunol 2011;128:896-897.

11. Bonini S, Lambiase A, Marchi S, Marchi S, Pasqualetti P, Zuccaro $O$, et al. Vernal keratoconjunctivitis revisited: a case series of 195 patients with long-term followup. Ophthalmology 2000;107:1157-1163.

12. Leonardi A. Management of Vernal Keratoconjunctivitis. Ophthalmol Ther 2013;2:73-88.

13. Leonardi A, Bogacka E, Fauquert JL, Leonardi A, Bogacka E, Fauquert $\mathrm{J}$, et al. Ocular allergy: recognizing and diagnosing hypersensitivity disorders of the ocular surface. Allergy 2012;67:1327-37.

14. Leonardi A. Vernal keratoconjunctivitis: pathogenesis and treatment. Prog Retin Eye Res 2002;21:319-39.

15. McGhee CN, Dean S, Danesh-Meyer H. Locally administered ocular corticosteroids: benefits and risks. Drug Saf 2002;25:3355.

16. Ang M, Ti SE, Loh R, Ang M, Ti SE, Loh R, et al. Steroid-induced ocular hypertension in Asian children with severe vernal keratoconjunctivitis. Clin Ophthalmol 2012;6:1253-8.

17. Utine CA, Stern M, Akpek EK. Clinical review: topical ophthalmic use of cyclosporin A. Ocul Immunol Inflamm 2010;18:352-61.

18. Cetinkaya A, Akova YA, Dursun D, Pelit A. Topical cyclosporine in the management of shield ulcers. Cornea. 2004;23:194-200.

19. Daniell M, Constantinou M, Vu HT, Taylor HR. Randomised controlled trial of topical ciclosporin $\mathrm{A}$ in steroid dependent allergic conjunctivitis. Br J Ophthalmol 2006;90:461-4.

20. Ozcan AA, Ersoz TR, Dulger E.Management of severe allergic conjunctivitis with topical cyclosporin a $0.05 \%$ eyedrops. Cornea 2007;26:1035-8.

21. Keklikci U, Dursun B, Cingu A. Topical Cyclosporine A $0.05 \%$ Eyedrops in the Treatment of Vernal Keratoconjunctivitis Randomized Placebo-Controlled Trial*. Adv Clin Exp Med 2014;23:1899-5276.

22. Un E, Sener B. Topical $0.05 \%$ cyclosporin A therapy in children with severe vernal keratoconjunctivitis. İzmir Dr. Behçet Uz Çocuk Hast. Dergisi. 2013;3:33-37.

23. Wan $\mathrm{KH}$, Chen $\mathrm{L}$, Rong SS, Pang CP, Young AL. Topical Cyclosporine in the Treatment of Allergic Conjunctivitis: A Meta-analysis. Ophthalmology 2013;120:2197-203

24. Tang-Liu DD, Acheampong A. Ocular pharmacokinetics and safety of ciclosporin, a novel topical treatment for dry eye. Clin Pharmacokinet 2005; 44:247-61

25. Acheampong AA, Shackleton M, Tang-Liu DD, Ding S, Stern $M E$, Decker R. Distribution of cyclosporin A in ocular tissues after topical administration to albino rabbits and beagle dogs. Curr Eye Res 1999;18:91-103. 